\title{
Recuento leucocitario y riesgo cardiovascular tras un síndrome coronario agudo
}

\author{
JOSÉ LUIS CABRERIZO G. ${ }^{1}$, BEGOÑA ZALBA E. ${ }^{2}$, \\ JUAN I. PÉREZ C. ${ }^{1}$, FRANCISCO RUIZ R. ${ }^{3}$
}

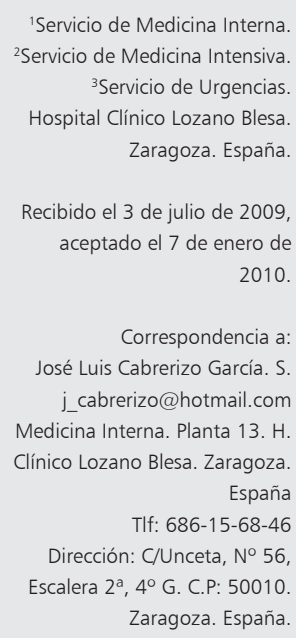

'Servicio de Medicina Interna. 2Servicio de Medicina Intensiva. ${ }^{3}$ Servicio de Urgencias. Hospital Clínico Lozano Blesa. Zaragoza. España.

Recibido el 3 de julio de 2009 aceptado el 7 de enero de 2010.

Correspondencia a: José Luis Cabrerizo García. S j_cabrerizo@hotmail.com Medicina Interna. Planta 13. H. Clínico Lozano Blesa. Zaragoza. España TIf: 686-15-68-46 Dirección: C/Unceta, № 56 Escalera 2a $4^{\circ}$ G. C.P: 50010 Zaragoza. España.

\section{Leukocyte count as a risk factor for coronary adverse events among patients admitted for an acute coronary syndrome}

\begin{abstract}
Background: Leukocytosis occurs as a response to a variety of inflammatory processes. Aim: To determine if white blood cell count (WBCC) at admission among patients who suffer an acute coronary syndrome (ACS) has an independent prognostic value for the occurrence of new cardiovascular adverse events (CAE). Patients and Methods: Prospective study of 558 patients aged $68 \pm 13$ years (122 women) admitted to the hospital for an acute coronary syndrome. WBCC was measured at admission. A logistic regression model was used to assess the association of WBCC with the occurrence of CAE during the next six months after admission (post infarction angina, re-infarction, cardiac failure and mortality). Results: An univariate analysis showed that patients with a WBCC $>15.000 \mathrm{cell} / \mathrm{mm}^{3}$ had a significantly higher mortality and occurrence of CAE. The multivariate analysis showed that subjects with WBCC $<10.000 \mathrm{cell} / \mathrm{mm}^{3}$ experienced fewer CAE than subjects with a WBCC $>15.000 \mathrm{cell} / \mathrm{mm}^{3}$, with an odds ratio of 0.46 (95\% confidence intervals $=0.21-0.97$, $p=0,042$ ). Conclusions: An elevated WBCC at admission among patients with an $A C S$ is associated with a higher incidence of CAE in the ensuing six months.

(Rev Med Chile 2010; 138: 274-280).
\end{abstract}

Key words: Acute coronary syndrome; Leucocyte count; Prognosis.
$\mathrm{E}$ 1 síndrome coronario agudo (SCA) constituye una de las formas más graves de la enfermedad coronaria y supone la causa más frecuente de morbimortalidad en Occidente. Los pacientes con SCA, en especial sin elevación del segmento ST (SCASEST) son un grupo con marcada variabilidad clínica y fisiopatológica, lo que condicionan un pronóstico diferente. En los últimos años se han desarrollado algoritmos para tratar de predecir su pronóstico y dirigir el tratamiento según el riesgo individual: los más conocidos son GRACE, PREDICT, TIMI, InTIME, CPP, GUSTO y PURSUIT. Sin embargo, muchos de ellos no son aplicables a la población general con elevada comorbilidad y no tienen en cuenta aspectos importantes como el papel de la inflamación en el desarrollo de la aterosclerosis y la precipitación de eventos cardiovasculares ${ }^{1}$. Según la teoría de "respuesta al daño" propuesta por Ross, la aterosclerosis es el resultado de una respuesta exagerada de tipo inflamatorio-fibroproliferativa que no sólo promueve el inicio del proceso aterosclerótico, sino que también contribuye al posterior crecimiento del ateroma y a la precipitación de sucesos trombóticos agudos ${ }^{2,3}$. Recientemente se ha destacado el papel de ciertos marcadores inflamatorios en el pronóstico de pacientes con SCA como la proteína plasmática A asociada al emba- 
razo (PAPP-A), la mioloperoxidasa, la proteína sérica amiloide, el sCD40-ligando o la albúmina modificada por isquemia ${ }^{4}$. Pero también está ampliamente reconocido el papel de reactantes de fase aguda como la PCRus, el fibrinógeno, la velocidad de sedimentación globular o el recuento leucocitario (RL). Durante el último medio siglo se ha observado la asociación entre leucocitosis y el pronóstico de la enfermedad cardiovascular ${ }^{5}$. El mecanismo fisiopatológico básico es doble alterando las propiedades hemorreológicas sanguíneas, por incremento de la viscosidad favoreciendo la obstrucción de pequeños vasos, y a través de la acción directa de enzimas proteolíticas mediante daño vascular directo y promoviendo la trombosis.

Nuestro objetivo fue determinar la posible relación entre el RL elevado en pacientes que sufren un SCA en una población no seleccionada, y su relación con el pronóstico en cuanto a nuevos eventos cardiovasculares adversos (ECVA) del tipo de angor-postinfarto, reinfarto e insuficiencia cardíaca, y con la mortalidad, independientemente de otros factores de riesgo.

\section{Pacientes y Método}

\section{Pacientes}

Se analizaron de forma prospectiva 589 pacientes que ingresaron consecutivamente entre enero de 2006 y diciembre de 2007 en nuestro hospital con sospecha de infarto agudo de miocardio (IAM), tanto con elevación como sin elevación del segmento ST según los criterios diagnósticos de la ACA/AHA (American College of Cardiology/ American Heart Association) ${ }^{6,7}$. Se excluyeron aquellos que no cumplían dichos criterios, los que no contaban con la determinación, aquellos que presentaran neoplasia maligna de órgano sólido o hematológica, síndrome febril o sepsis en el momento del estudio, enfermedades autoinmunes o inflamatorias, insuficiencia renal crónica en estadio 5 según las guías K/DOQI 2002 de la National Kidney Foundation ${ }^{8}$ o imposibilidad de conocer la evolución durante los meses de seguimiento. Finalmente, 558 pacientes quedaron incluidos en el estudio. La terapéutica aplicada y la realización de estudio invasivo y revascularización se realizó de acuerdo con las guías de práctica clínica.

Variables incluidas en el estudio: Las variables estimadas fueron: Estratificación de riesgo según las escala GRACE (GRACE alto, GRACE medio y GRACE bajo), edad, sexo, peso, hipertensión arterial, diabetes, dislipemia, tabaquismo, angor previo, insuficiencia cardíaca previa, infarto previo, descenso del segmento ST, frecuencia cardíaca, tensión arterial sistólica, tensión arterial diastólica, presión de pulso, enzimas cardíacas elevadas (CK, CK-MB o ambos), troponina I elevada, presencia de arritmias, parada cardiorrespiratoria recuperada, fracción de eyección y las siguientes variables analíticas dentro de las primeras tres horas del ingreso: hemoglobina, glucemia, natremia, kaliemia, concentraciones de fibrinógeno, función renal mediante índice de Cockcroft y recuento leucocitario.

Definición de eventos y seguimiento: Se definen como eventos principales la muerte por cualquier causa, y eventos cardiovasculares adversos en caso de angor-postinfarto, reinfarto ${ }^{9}$ o insuficiencia cardíaca $^{10}$ en el período de seguimiento de seis meses desde el momento del ingreso mediante revisión de historia clínica o contacto telefónico.

Estadística: La medición del RL al ingreso fue categorizado en tres grupos. grupo 1: $<10.000$ cell/ $\mathrm{mm}^{3}$; grupo $2: 10.000-15.000 \mathrm{cell} / \mathrm{mm}^{3}$ y grupo 3 : $>15.000 \mathrm{cell} / \mathrm{mm}^{3}$, teniendo en cuenta puntos de corte de trabajos previos ${ }^{11,12}$.

Se fijó un valor de $\mathrm{p}=0,05$ para aceptar o rechazar hipótesis. La mortalidad acumulada para cada grupo del RL se presenta mediante las curvas de Kaplan-Meier con el estadístico de Log-Rank. Se realizó un análisis de regresión logística inicialmente bivariante y posteriormente multivariante, incluyendo aquellas variables con una $\mathrm{p}<0,16$ en el análisis bivariado previo. Los coeficientes estimados fueron expresados como odds ratio con sus respectivos intervalos de confianza del 95\% (IC del 95\%). Los valores se consideraron significativos para $\mathrm{p}<0,05$. Para el análisis estadístico se utilizó el programa SPSS 12.0

\section{Resultados}

Las caractarísticas de la población estudiada se muestran en la Tabla 1. La media del recuento leucocitario fue $11.271,7 \mathrm{cell} / \mathrm{mm}^{3}$ con una DE de $4.152,2 \mathrm{cell} / \mathrm{mm}^{3}$ y una mediana de 10.400 cell/ $\mathrm{mm}^{3}$. Según la estratificación de riesgo GRACE, la mayor parte de la muestra pertenecía al grupo de riego bajo: 265 pacientes (48,2), 177 al grupo de riesgo medio $(32,2 \%)$ y 108 al grupo de alto riesgo $(19,6 \%)$. La media de supervivencia durante los 
Tabla 1. Características basales de la muestra

\begin{tabular}{|c|c|c|c|c|c|}
\hline Variable & $\mathbf{n}$ & $\%$ & Variable & $\mathbf{n}$ & $\%$ \\
\hline Sexo mujer & 122 & 21,8 & V. Analítica(a) & & \\
\hline Peso $(k g)^{(a)}$ & $78,2 \pm 47$ & & Hemoglobina (g/dl) & $14,1 \pm 1,9$ & \\
\hline Edad (años) $)^{(a)}$ & $68 \pm 12,7$ & & Recuento leucocitario (cell/ $/ \mathrm{mm}^{3}$ ) & $11.271,7 \pm 4.1252,2$ & \\
\hline FRCV & & & Glucemia (mg/dl) & $165 \pm 83,7$ & \\
\hline Hipertensión & 310 & 55,5 & Sodio (Emq/L) & $137,8 \pm 3,2$ & \\
\hline Diabetes & 122 & 21,8 & Potasio (Emq/L) & $4,2 \pm 2,5$ & \\
\hline Dislipemia & 256 & 45,8 & Fibrinógeno (mg/dl) & $423,9 \pm 143$ & \\
\hline Tabaquismo & 243 & 43,5 & Índice de Cockcroft (ml/min) & $81,5 \pm 35,1$ & \\
\hline Antecedentes & & & Arritmias & 133 & 23,8 \\
\hline Angor previo & 163 & 29,2 & Parada cardiorrespiratoria & 15 & 2,7 \\
\hline Insuficiencia cardíaca previa & 24 & 4,3 & Mortalidad & 64 & 11,4 \\
\hline Infarto previo & 84 & 15 & Shock cardiogénico & 41 & 64,1 \\
\hline Segmento ST & & & Rotura cardíaca & 6 & 9,4 \\
\hline Elevado & 351 & 62,8 & Fibrilación ventricular & 4 & 6,3 \\
\hline Descendido & 119 & 21,3 & Parada cardiorrespiratoria & 4 & 6,3 \\
\hline Isoeléctrico & 89 & 15,9 & Accidente cerebrovascular & 4 & 6,3 \\
\hline Enzimas cardíacas & 456 & 81,6 & IAM & 1 & 1,5 \\
\hline Troponina I & 550 & 98,4 & Otras & 4 & 6,3 \\
\hline Fracción de eyección & & & ECVAS & 129 & 23,1 \\
\hline$>50 \%$ & 317 & 64,2 & Angor post-infarto & 77 & 14,3 \\
\hline $40-50 \%$ & 97 & 19,6 & Reinfarto & 26 & 4,8 \\
\hline $30-40 \%$ & 56 & 11,3 & Insuficiencia cardíaca & 26 & 4,8 \\
\hline$<30 \%$ & 24 & 4,9 & & & \\
\hline \multicolumn{6}{|l|}{ Escala GRACE } \\
\hline Riesgo bajo & 265 & 48,2 & & & \\
\hline Riesgo medio & 177 & 32,2 & & & \\
\hline Riesgo alto & 108 & 19,6 & & & \\
\hline
\end{tabular}

(a)Media \pm desviación estándar. FRCV: Factores de riesgo cardiovascular V: variables. ECVAS: Eventos cardiovasculares adversos.

seis meses de seguimiento fue de 162,7 días (IC $95 \%$ 158,8-166,91). Se registraron 64 fallecimientos (11,4\%); las causas más frecuentes fueron: 41 casos de shock cardiogénico; $(64,1 \%)$ seguido de 6 roturas cardíacas $(9,4 \%), 4$ casos de fibrilación ventricular $(6,3 \%), 4$ paradas cardiorrespiratorias (6,3\%), 4 accidentes cerebrovasculares y 1 caso IAM ( $1,5 \%)$; todos los episodios con resultado final de muerte. Ocurrieron 129 eventos cardiovasculares adversos $(23,1 \%)$ : 77 casos de angor post-infarto (14,3\%), 26 reinfartos $(4,8 \%)$ y 26 casos de insuficiencia cardiaca $(4,8 \%)$.
Los valores medios del RL al ingreso eran significativamente mayores en los pacientes que presentaban eventos o fallecían en el período de seguimiento. $12.445 \pm 5.059 \mathrm{cell} / \mathrm{mm}^{3}$ en el grupo de fallecidos y $11.120 \pm 4.000 \mathrm{cell} / \mathrm{mm}^{3}$ en el de supervivientes $(\mathrm{p}<0,001) \cdot 12.682 \pm 5.144 \mathrm{cell} / \mathrm{mm}^{3}$ entre los que presentaban ECVA y $10.865 \pm 3.727$ cell $/ \mathrm{mm}^{3}$ entre los que no ( $\left.\mathrm{p}<0,001\right)$, Figura 1.

Análisis univariante: La mortalidad y ECVA fueron significativamente más frecuentes en el grupo de pacientes con RL mayor, Figura 2. En relación a la mortalidad, en el grupo 1: fallecieron 


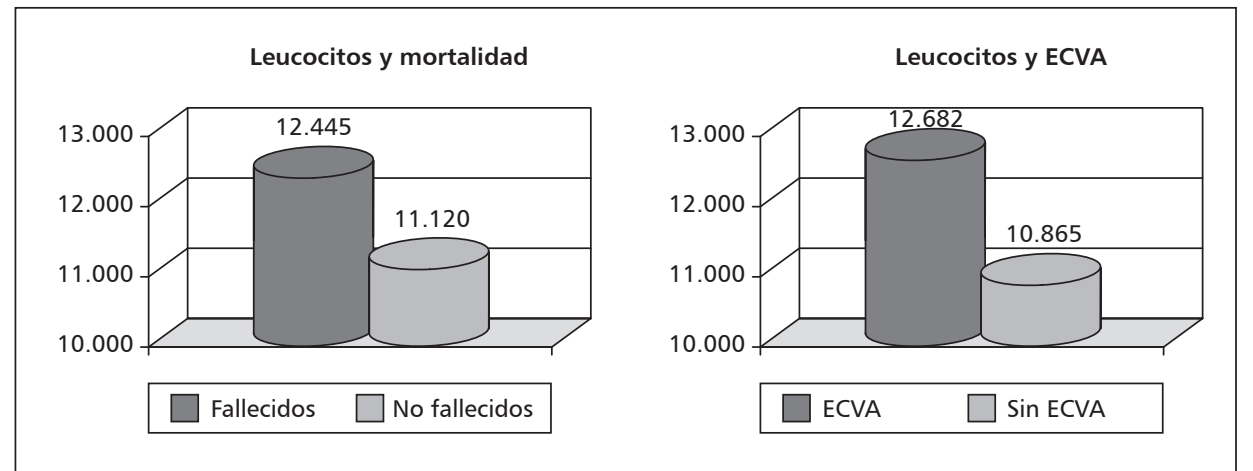

Figura 1. Medias del recuento leucocitario al ingreso.
20 pacientes $(8,2 \%)$, en el grupo $2: 28(12,2 \%)$ y en el grupo 3: $16(18,8 \%), p=0,028$. En relación a los ECVAs: en el grupo 1: 40 (16,5\%) presentaban alguno de ellos, en el grupo 2: $54(23,5 \%)$ y en el grupo 3: $31(36,5 \%), p=0,001$, Tabla 2 . La supervivencia acumulada de cada uno de los tres grupos se puede observar en la curva de Kaplan Meier, Figura 3.

La Tabla 2 recoge las variables que presentaban relación más directa con la frecuencia de eventos cardiovasculares adversos.

Análisis multivariante: Con intención de analizar la relación independiente del RL al ingreso y los EVCAS se efectuó el análisis multivariante incluyendo las siguientes variables: edad, sexo, peso, tabaquismo, angor previo, insuficiencia cardíaca previa, infarto previo, frecuencia cardíaca, tensión arterial diastólica, enzimas cardíacas elevadas, presencia de arritmias, parada cardiorrespiratoria recuperada, RL por grupos, glucemia $>160 \mathrm{mgrs} / \mathrm{dl}$, fibrinógeno $>450 \mathrm{mg} / \mathrm{dl}$, e índice de Cockcroft $<$ $160 \mathrm{ml} / \mathrm{min}$. Los pacientes con RL menor a 10.000 cell $/ \mathrm{mm}^{3}$ al ingreso presentaban una probabilidad

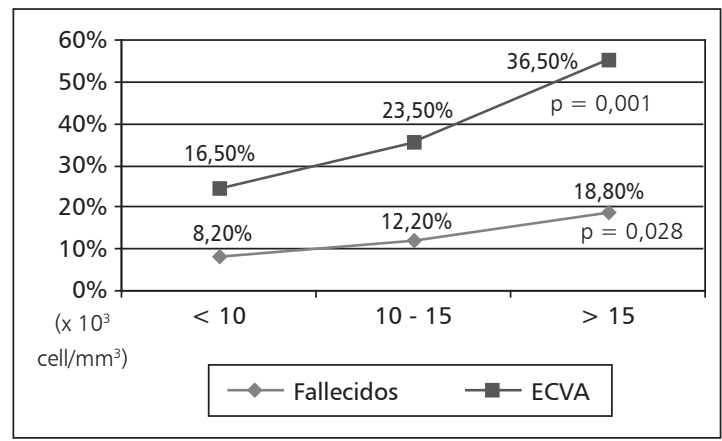

Figura 2. Leucocitos, ECVA y mortalidad. de eventos que es $45 \%$ menor a los pacientes con recuento $>15.000 \mathrm{cell} / \mathrm{mm}^{3}$. La OR para el grupo $1:<10,000$ cell $/ \mathrm{mm}^{3}$ fue de $0,456, p=0,042$ (IC $95 \%, 0,214-0,974)$, es decir, pertenecer al grupo con RL menor protegía frente a la presencia de eventos cardiovascualares adversos Tabla 3.

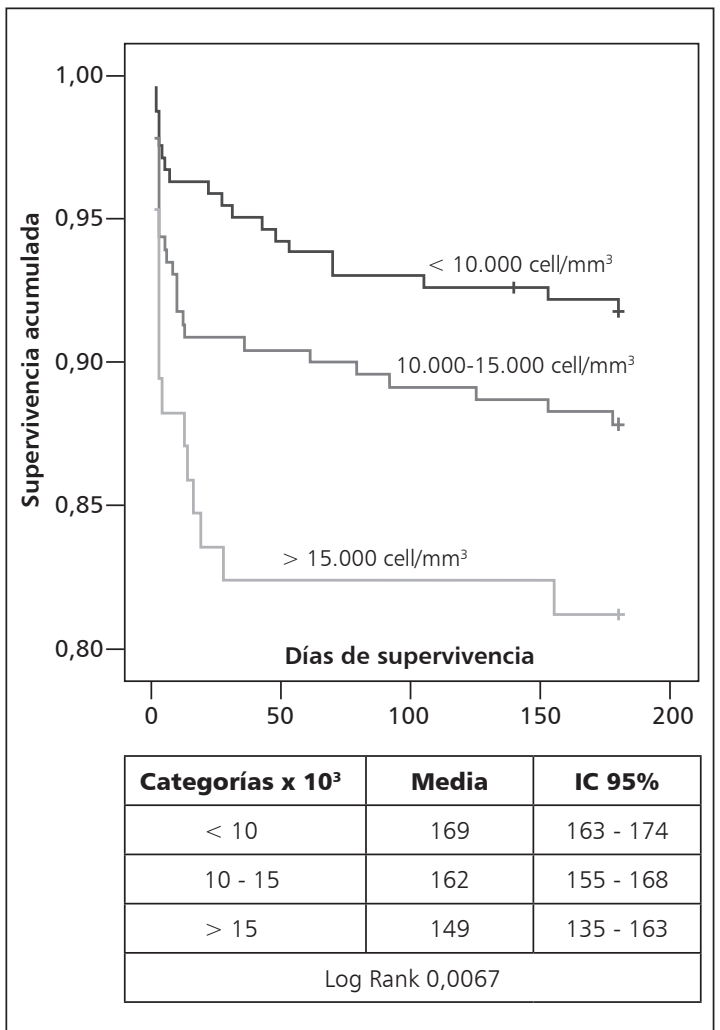

Figura 3. Función de supervivencia del recuento leucocitario. 
Tabla 2. Predictores pronósticos de eventos cardiovasculares adversos a seis meses. Análisis bivariante

\begin{tabular}{|c|c|c|c|}
\hline Variable & $\begin{array}{c}\text { Eventos CVA } \\
(n=129)\end{array}$ & $\begin{array}{l}\text { No Eventos CVA } \\
\quad(n=430)\end{array}$ & $\mathbf{p}$ \\
\hline GRACE de alto riesgo & $38,9 \%$ & $61,1 \%$ & $<0,001$ \\
\hline Edad $>80$ años & $33,3 \%$ & $66,7 \%$ & 0,001 \\
\hline Sexo mujer & $35,2 \%$ & $64,8 \%$ & $<0,001$ \\
\hline Peso $(k g)^{(a)}$ & $75 \pm 14,5$ & $79 \pm 14,7$ & 0,01 \\
\hline Tabaquismo & $18,9 \%$ & $81,1 \%$ & 0,073 \\
\hline Angor previo & $27 \%$ & $73 \%$ & 0,106 \\
\hline Insuficiencia cardíaca previa & $45,8 \%$ & $54,2 \%$ & 0,005 \\
\hline Infarto previo & $29,8 \%$ & $70,2 \%$ & 0,086 \\
\hline Descenso del segmento ST & $28,6 \%$ & $71,4 \%$ & 0,204 \\
\hline Frecuencia cardíaca(a) & $82,5 \pm 22,9$ & $75,8 \pm 19,6$ & 0,002 \\
\hline Tensión arterial sistólica(a) & $133,2 \pm 30,9$ & $135,5 \pm 26,7$ & 0,216 \\
\hline Tensión arterial diastólica(a) & $75,8 \pm 19,3$ & $79,4 \pm 17,2$ & 0,034 \\
\hline Enzimas cardíacas elevadas & $24,3 \%$ & $75,7 \%$ & 0,043 \\
\hline Arritmias & $27,1 \%$ & $72,9 \%$ & 0,152 \\
\hline Parada cardiorrespiratoria recuperada & $46,7 \%$ & $53,3 \%$ & 0,052 \\
\hline Glucemia > $160 \mathrm{mgr} / \mathrm{dl}$ & $32,5 \%$ & $67,5 \%$ & $<0,001$ \\
\hline Fibrinógeno > 450 mg/dl & $26,8 \%$ & $73,2 \%$ & 0,101 \\
\hline Índice de Cockcroft $<60 \mathrm{ml} / \mathrm{min}$ & $31,9 \%$ & $68,1 \%$ & $<0,001$ \\
\hline Categorías del recuento leucocitario (cell/mm³) & & & 0,001 \\
\hline Grupo 1: < 10.000 & $16,5 \%$ & $83,5 \%$ & \\
\hline Grupo 2: $10.000-14.900$ & $23,5 \%$ & $76,5 \%$ & \\
\hline Grupo 3: > 15.000 & $36,5 \%$ & $63,5 \%$ & \\
\hline
\end{tabular}

(a)Media \pm desviación estándar.

Tabla 3. Recuento leucocitario y eventos cardiovasculares adversos a seis meses. Análisis multivariante

\begin{tabular}{|lcccc|}
\hline Variable & p & OR & \multicolumn{2}{c|}{ IC al 95\% } \\
Inferior & Superior \\
$\begin{array}{l}\text { Recuento } \\
\text { leucocitario } \\
<10.000 \\
\text { cell/ } \mathrm{mm}^{3}\end{array}$ & 0,042 & 0,456 & 0,214 & 0,974 \\
\hline
\end{tabular}

\section{Discusión}

En el estudio observamos una relación entre el RL elevado al ingreso tras un SCA y un peor pronóstico en los siguientes seis meses.

El porcentaje de fallecidos con $\mathrm{RL}>15.000$ cell $/ \mathrm{mm}^{3}(18,8 \%)$ era más del doble que aquellos con $\mathrm{RL}<10.000$ cell $/ \mathrm{mm}^{3}(8,2 \%)$. Dos grandes estudios reflejan esta relación. En el caso de Barron et $\mathrm{al}^{13}$, observan en 153.213 pacientes mayores de 65 que un $\mathrm{RL}>13.600 \mathrm{cell} / \mathrm{mm}^{3}$ en las primeras 24 horas tras sufrir un IAM se comporta como factor independiente de mortalidad dentro de los primeros 30 días tras el ingreso y de eventos adversos intrahospitalarios. Grzybowski M, et al ${ }^{14}$, obtienen conclusiones similares en 115.273 pacientes independientemente de la edad. También se ha observado esta relación para períodos más largos de seguimiento y pacientes sin elevación del segmento $\mathrm{ST}^{15}$.

Asimismo, parece haber un paralelismo con la presencia de eventos cardiovasculares adversos. En la muestra pudimos comprobar esta relación independientemente de otras variables de riesgo conocidas, de tal manera que los pacientes con $\mathrm{RL}<10.000$ cell $/ \mathrm{mm}^{3}$ sufrían menor número de 
anginas post-infarto, reinfartos o episodios de insuficiencia cardíaca en los siguientes seis meses. Algunos trabajos coinciden con estos resultados, sobre todo en el caso de la insuficiencia cardíaca y el shock cardiogénico ${ }^{16,17}$.

Estos episodios están en relación con el proceso inflamatorio y de hipercoagulabilidad que en último término incrementa el área de isquemia miocárdica, más que a través del daño celular directo. Recientemente, Engström et al ${ }^{18}$ han realizado un estudio poblacional de 16.940 pacientes sin antecedentes de infarto, comprobando que ante mayores recuentos leucocitarios aumentaban las hospitalizaciones por insuficiencia cardíaca. Parece tan establecida la relación entre el RL y el pronóstico en la cardiopatía isquémica que incluso se ha observado relación con el tamaño del infarto, la fracción de eyección (FE), la prevalencia de la enfermedad multivaso y el éxito tras la revascularización ${ }^{19}$. En nuestros resultados también observamos una relación significativa entre RL mayor y una menor FE, de tal manera que en el grupo 3, 9,6\% presentaban un $\mathrm{FE}<30 \%$ en comparación con el grupo 2: 5,8\% o el grupo $1: 2,3 \%(\mathrm{p}=0,011)$.

La trombosis y la inflamación forman parte de las cinco causas que pueden desestabilizar, según Braunwald ${ }^{20}$, la placa de ateroma y precipitar un evento coronario agudo. La leucocitosis en el SCA es un marcador que podría reflejar el estado inflamatorio presente en el proceso aterogénico a lo largo de su historia natural. En este sentido, podría favorecer la hipercoagulabilidad y el estado protrombótico aumentando la viscosidad sanguínea y modificando sus propiedades hemorreológicas, pero también por incremento del factor tisular, una mayor activación plaquetaria, alteraciones del fibrinógeno, y aumentado los agregados circulantes con efecto predominantemente sobre la microcirculación. También ejerce un efecto vascular a través de radicales superóxido, citocinas o mieloperoxidasas, y acelera la aterosclerosis oxidando lipoproteínas, incrementando el infiltrado monocitario, etc ${ }^{21}$.

En los últimos años se ha investigado sobre un gran número de nuevos biomarcadores que se puedan usar junto con los ya establecidos, con el fin de explorar su utilidad como herramientas diagnósticas y de estratificación de riesgo ${ }^{22}$, sin embargo, los marcadores de inflamación no son empleados de forma rutinaria en el entorno clínico.

\section{Limitaciones}

Siendo este estudio observacional no podemos establecer una aleatorización estratificada, sin embargo, los resultados obtenidos se aproximan a la realidad de la práctica habitual en nuestro medio sin selección previa de los casos. A pesar de un tamaño muestral correcto, los datos son de un sólo centro y de éstos se perdieron algunos casos. Esto puede afectar a la validez externa. Algunos de los trabajos con los que hemos comparado nuestros resultados habían utilizado diferentes puntos de corte para la estratificación de las variables dificultado las comparaciones. No obstante, todos apuntan en la misma dirección. Hemos asumido que los tratamientos empleados eran los recomendados por las guías de práctica clínica. Variaciones en pacientes similares han podido modificar su pronóstico. El seguimiento se realizó durante 6 meses, período limitado que de haber sido superior aportaría más información a largo plazo.

\section{Conclusiones}

Nuestros resultados sugieren que el recuento leucocitario puede ser útil en la identificación de grupos de riesgo tras un IAM con y sin elevación del segmento ST. Refleja la intensidad del sustrato inflamatorio que acompaña a la enfermedad coronaria desde su génesis hasta la precipitación de eventos agudos. Determinación, por otra parte, barata, fiable, fácil de interpretar y presente en toda determinación analítica. Sin embargo, se precisan nuevos estudios que permitan identificar su riego a nivel individual.

\section{Referencias}

1. Sanz GA. Estratificación del riesgo en los síndromes coronarios agudos: un problema no resuelto. Rev Esp Cardiol 2007; 60: 23-30.

2. Ross R. Atherosclerosis: an inflammatory disease. N Engl J Med 1999; 340: 115-26.

3. Libby P, Ridker PM, Maseri A. Inflammation and atherosclerosis. Circulation 2002; 105: 1135-43.

4. Bodí V, Fácila L, Sanchís J, Llácer A, Núñez J, Mainar L, et al. Pronóstico a corto plazo de los pacientes ingresados por probable síndrome coronario agudo sin elevación del segmento ST. Papel de los nuevos marcadores de daño miocárdico y de los reactantes de fase aguda. Rev Esp Cardiol 2002; 55: 823-30. 
5. Cole DR, Singian EB, Katz LN. The long-term prognosis following myocardial infarction, and some factors which affect it. Circulation 1954; 9: 321-34.

6. Anderson JL, Adams CD, Antman EM, Bridges CR, Califf RM, Casey de JR, et al. ACC/AHA 2007 guidelines for the management of patients with unstable angina/ non ST-elevation myocardial infarction: a report of the American College of Cardiology/American Heart Association Task Force on Practice Guidelines (Writing Committee to Revise the 2002 Guidelines for the Management of Patients With Unstable Angina/Non ST-Elevation Myocardial Infarction): developed in collaboration with the American College of Emergency Physicians, the Society for Cardiovascular Angiography and Interventions, and the Society of Thoracic Surgeons: endorsed by the American Association of Cardiovascular and Pulmonary Rehabilitation and the Society for Academic Emergency Medicine. Circulation 2008 Mar 4; 117 (9): e180.

7. Antman EM, Anbe DT, Armstrong PW, Bates ER, Green LA, Hand M, et al. ACC/AHA guidelines for the management of patients with ST-elevation myocardial infarction: a report of the American College of Cardiology/American Heart Association Task Force on Practice Guidelines (Committee to Revise the 1999 Guidelines for the Management of Patients with Acute Myocardial Infarction). Ciculation 2004 Aug 31; 110(9): e82-292.

8. National Kidney Foundation. K/DOQI clinical practice guidelines for chronic kidney disease: evaluation, classification, and stratification. Am J Kidney Dis 2002 Feb; 39 (2 Suppl 1): S1-266.

9. Arós F, Loma-Osorio Á, Alonso A, Alonso JJ, Cabadés A, Coma-Canella I, et al. Guías de actuación clínica de la Sociedad Española de Cardiología en el infarto agudo de miocardio. Rev Esp Cardiol 1999; 52: 919-56.

10. Nieminen MS, Böhm M, Cowie MR, Dressler H, Filippatos GS, Jondeau G, et al. Guías de Práctica Clínica sobre el diagnóstico y tratamiento de la insuficiencia cardíaca aguda. Versión resumida. Rev Esp Cardiol 2005; 58: 389429.

11. Barron HV, Cannon CP, Murphy SA, Braunwald E, Gibson CM. Association between white blood cell count, epicardial blood flow, myocardial perfusion, and clinical outcomes in the setting of acute myocardial infarction: a thrombolysis in myocardial infarction 10 substudy. Circulation 2000; 102: 2329-34.

12. Bhatt DL, Chew DP, Lincoff AM, Simoons ML, Harrington RA, Ommen SR, et al. Effect of revascularization on mortality associated with an elevated white blood cell count in acute coronary syndromes. Am J Cardiol 2003; 92: 136-40.

13. Barron HV, Harr SD, Radford MJ, Wang Y, Krumholz HM. The association between white blood cell count and acute myocardial infarction mortality in patients $>$ or $=65$ years of age: findings from the cooperative cardiovascular project. J Am Coll Cardiol 2001; 38: 1654-61.

14. Grzybowski M, Welch Rd, Parsons L, Ndumele Ce, Chen E, Zalenski R, et al. The association between white blood cell count and acute myocardial infarction in-hospital mortality: findings from the National Registry of Myocardial Infarction. Acad Emerg Med 2004; 11: 1049-60.

15. Sabatine MS, Morrow DA, Cannon CP, Murphy SA, Demopoulos LA, Dibattiste PM, et al. Relationship between baseline white blood cell count and degree of coronary artery disease and mortality in patients with acute coronary syndromes: a TACTICS-TIMI 18 (Treat Angina with Aggrastat and determine Cost of Therapy with an Invasive or Conservative Strategy-Thrombolysis in Myocardial Infarction 18 trial) substudy. J Am Coll Cardiol 2002 20; 40: 1761-8.

16. Furman MI, Gore JM, Anderson FA, Budaj A, Goodman SG, Avezum A, et al. Elevated leukocyte count and adverse hospital events in patients with acute coronary syndromes: findings from the Global Registry of Acute Coronary Events (GRACE). Am Heart J 2004; 147: 42-8.

17. Menon V, Lessard D, Yarzebski J, Furman MI, Gore JM, Goldberg RJ. Leukocytosis and adverse hospital outcomes after acute myocardial infarction. Am J Cardiol 2003 15; 92: 368-72.

18. Engström G, Melander O, Hedblad B. Leukocyte count and incidence of hospitalizations due to heart failure: A population-based cohort study. Circulation: Heart Failure. Published Online on March 23, 2009.

19. Turner SJ, Ketch TR, Gandhi SK, Sane DC. Routine hematologic clinical tests as prognostic markers in patients with acute coronary syndromes. Am Heart J 2008; 155 : 806-16.

20. Braunwald E. Unstable angina: An etiologic approach to management. Circulation 1998; 98: 2219-22.

21. Coller BS. Leukocytosis and ischemic vascular disease morbidity and mortality: is it time to intervene? Arterioscler Thromb Vasc Biol 2005; 25: 658-70. Epub 2005 Jan 20.

22. Bassand JP, Hamm CW, Ardissino D, Boersma E, Budaj A, Fernández-Avilés F, et al. Guía de Práctica Clínica para el diagnóstico y tratamiento del síndrome coronario agudo sin elevación del segmento ST. Rev Esp Cardiol 2007; 60: 1070.e1-e80. 